\title{
Formal linguistic theories
}

\section{SUSANNE ANNIKKI KRISTENSEN}

In this article, formal grammar is considered to contrast with descriptive grammar much more than with functional grammar. Following Stuurmann 1996, the article subscribes to the view that "descriptive grammar comprehensively includes facts whereas formal grammar is exclusively directed at the underlying system". It is presupposed that generative and (most) functional theories are formal theories because they are directed at the underlying system, whereas e.g. Hopper's Emergent grammar, which does not recognize the existence of an underlying system, is considered to be descriptive. Two generative theories, namely Chomsky's Generativism and Lexical-Functional Grammar, are compared to two functional theories, namely Functional Grammar and Functional Syntax, and the article's overall goal is to point out differences and, more importantly, similarities between the generative and functional theories of syntax. 